\title{
Monoclonal Antibody HeFi-1
}

National Cancer Institute

\section{Source}

National Cancer Institute. Monoclonal Antibody HeFi-1. NCI Thesaurus. Code C2505.

A murine monoclonal antibody with potential antineoplastic activity. Monoclonal antibody HeFi-1 binds to CD30, a cell surface antigen found on mitogen-activated B-cells and Tcells, and Reed-Sternberg cells. Monoclonal antibody HeFi-1 has been shown to arrest tumor growth and prevent metastasis in animal models. ( $\mathrm{NCl04)}$ 